\title{
Two-bunch self-seeding for narrow-bandwidth hard x-ray free-electron lasers
}

\author{
Yuantao Ding, Zhirong Huang, and Ronald D. Ruth \\ SLAC National Accelerator Laboratory, Menlo Park, California 94025, USA
}

(Received 22 March 2010; published 30 June 2010)

\begin{abstract}
It is well known that seeding can be used to produce narrow-bandwidth and fully coherent x-ray freeelectron lasers (FELs). Self-seeding, which uses an extra undulator to generate the seed pulse, is perhaps one of the most promising methods to accomplish this. In the hard x-ray regime with high-energy electrons, this method requires a large magnetic chicane to match the path-length delay of the $\mathrm{x}$-ray monochromator that selects a narrow bandwidth of radiation. Such a chicane not only takes a large footprint to build, but also may degrade the electron-beam qualities through incoherent and coherent synchrotron radiation. In this paper, we present an alternative two-bunch self-seeding scheme. The two bunches are precisely separated to match the x-ray delay of the monochromator and eliminate the need for a long, complex magnetic chicane. The spectrally filtered self-amplified spontaneous emission x-ray pulse produced by the first bunch is combined with the second electron bunch at the entrance of the second undulator and then amplified to the saturation level. We present start-to-end simulation results based on the linac coherent light source hard $\mathrm{x}$-ray FEL and show that this method can produce a nearly fully coherent $\mathrm{x}$-ray pulse at a few GW power level.
\end{abstract}

DOI: 10.1103/PhysRevSTAB.13.060703

PACS numbers: 41.60.Cr

\section{INTRODUCTION}

The successful commissioning and operation of the linac coherent light source (LCLS) [1] has demonstrated that the $\mathrm{x}$-ray free-electron laser (FEL) has come of age; these types of $\mathrm{x}$-ray sources are poised to revolutionize the ultrafast $\mathrm{x}$-ray sciences. The LCLS and other hard $\mathrm{x}$-ray FELs under construction are based on the principle of selfamplified spontaneous emission (SASE) [2-5]). Although SASE FELs have excellent transverse coherence, the temporal coherence is very limited because the FEL process starts from random shot noise. To improve the temporal coherence of SASE FELs, various types of seeding have been proposed over the past years. Since a proper coherent seed does not exist at $\mathrm{x}$-ray wavelengths, a seed laser at a much longer wavelength can be used to initiate the modulation which can be frequency upshifted through a process called high-gain harmonic generation [6]. Recently, a more efficient harmonic generation process called echo-enabled harmonic generation has been proposed $[7,8]$. These two laser seeding schemes are expected to be used in extreme ultraviolet and soft x-ray FEL facilities. Instead of seeding with an external laser, the self-seeding approach [9] makes use of an x-ray monochromator to spectrally filter SASE light produced from a first undulator, which can then be used to seed an unmodulated electron bunch passing through a second undulator to produce an amplified narrow-bandwidth $\mathrm{x}$-ray FEL pulse. In principle, the selfseeding scheme is applicable to arbitrary wavelengths including the hard $\mathrm{x}$-ray wavelength range $[10,11]$. Other methods to achieve full longitudinal coherence include the use of $\mathrm{x}$-ray cavities and multiple bunches of electrons to form either an $\mathrm{x}$-ray regenerative amplifier [12] or an X-ray FEL oscillator [13].
Among these seeding schemes, self-seeding is relatively straightforward to implement for a single-pass high-gain FEL. It consists of two sequential undulators, and an x-ray monochromator and electron bypass chicane located between them. After the first undulator, the x-ray beam enters the monochromator that spectrally filters the SASE radiation. A bypass chicane removes the electron density modulation and matches the $\mathrm{x}$-ray delay introduced by the monochromator. The electron beam can then amplify the narrow-bandwidth radiation to saturation in the second undulator.

Nevertheless, there are several technical challenges which must be addressed to realize the self-seeding. For Angstrom-wavelength FELs such as the LCLS, the x-ray monochromator that filters the SASE radiation also introduces a path-length delay of about $1 \mathrm{~cm}$. To delay a very high-energy (10-20 GeV) electron beam by this amount, a large magnetic chicane $40-50 \mathrm{~m}$ long is necessary $[10,11]$. Such a complex chicane not only takes a large footprint to build but also generates incoherent and coherent synchrotron radiation (CSR) that may degrade the beam quality of the short electron bunch. An example of CSR studies in the self-seeding scheme for the FLASH soft $x$-ray FEL is given in Ref. [14].

Motivated by these considerations, we propose an alternative two-bunch self-seeding scheme (see Fig. 1). The two bunches are precisely separated and matched to the xray delay of the monochromator which can be increased easily to allow for a convenient bunch spacing. The electron-beam chicane serves to avoid the x-ray optics of the $\mathrm{x}$-ray chicane and can be accomplished with very small offsets and modest magnets. The weak chicane still has a sufficient momentum compaction to smear out the 


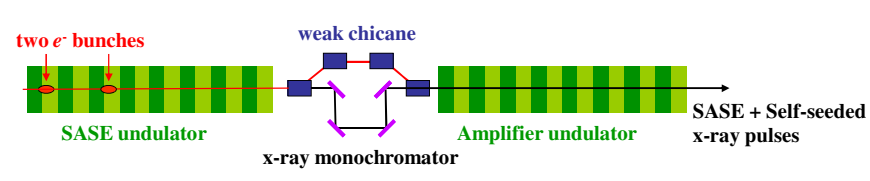

FIG. 1. (Color) Schematic of the two-bunch self-seeding scheme with a weak chicane and a crystal monochromator between two undulator sections.

Angstrom-scale microbunching of the electron beam. The spectrally filtered SASE light produced by the first bunch is combined with the second bunch at the entrance of the second undulator and can then be amplified to the saturation level. In Sec. II, we briefly describe methods and issues of generating two electron bunches with controllable delays. In Sec. III, we use an idealized bunch and a more realistic LCLS bunch to demonstrate the feasibility of the proposed scheme. ${ }^{1}$

\section{TWO-BUNCH GENERATION}

The key to making two-bunch self-seeding work is to produce two electron bunches at precise distances with respect to each other, and then to accelerate them and compress them so that the beam energy and position are nearly identical as they follow each other through both undulators. The most straightforward way to create two pulses which are identical but delayed with respect to each other is to split the ultraviolet laser pulse which illuminates the cathode, delay one of the pulses, and then recombine the pulses on the same trajectory heading towards the rf gun. One method for accomplishing this is shown in Fig. 2. A circularly polarized laser pulse is split in a polarizing beam splitter which transmits one linear polarization, but reflects the other polarization. These beams follow a path similar to that used for a Michelson interferometer except for two key differences: a quarter wave plate (QWP) is inserted into each path. This causes the linearly polarized light after retroreflection to be transmitted (reflected) in the reflected (transmitted) arms. In addition, one of the arms is longer than the other by one-half the desired total pathlength difference. Similar pulse stacking methods have been discussed in the literature [16].

The three-bunch mode was used in the SLAC linac to accelerate two bunches, electrons and positrons, for collisions at the SLAC linear collider, and a third electron bunch to create positions for the next cycle. Routine operation with $\sim 6 \mathrm{nC}$ per bunch and $\sim 60 \mathrm{~ns}$ spacing was achieved [17]. For the two-bunch self-seeding discussed here, we choose the separation of $10 \mathrm{~ns}$ (30 S-band rf periods) for LCLS. A smaller separation can save space for the x-ray monochromator, but the wakefields in the normal-conducting rf structure may affect the beam quality

\footnotetext{
${ }^{1}$ Just prior to the submission of this paper, we noticed a preprint that independently proposes a similar two-bunch selfseeding scheme [15].
}

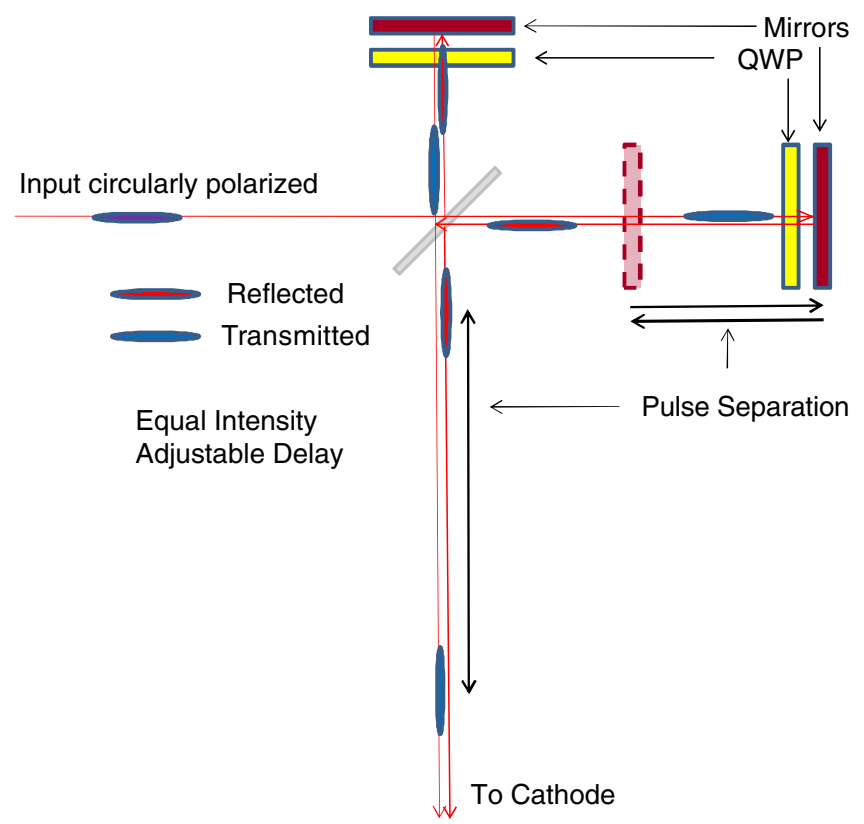

FIG. 2. (Color) Schematic of a method to produce two laser pulses that creates two electron bunches with a variable delay on the photocathode.

of the second bunch, and will be more challenging for beam diagnostics. With the LCLS beam parameters, the beam loading of the linac rf is less than $0.1 \%$, which will require an essentially flat $\mathrm{rf}$ amplitude between bunches. The estimated long-range wakefield effects with $10 \mathrm{~ns}$ spacing are very small for LCLS operating charges (between 250 and $20 \mathrm{pC}$ ); however, careful simulation studies will be required for this specific implementation. In passing, we note that the two-bunch operation mode with $\sim 10$ ns spacing has been demonstrated in the superconducting linac at FLASH [18]. With the superconducting rf cavity, the wakefield effects are much weaker; hence, the separation between the two bunches may be as short as one rf cycle [15].

\section{FEL SIMULATIONS}

We use GENESIS FEL code [19] with the LCLS hard Xray beam parameters to demonstrate the feasibility of the two-bunch self-seeding method. The main parameters used in the simulations are based on the LCLS commissioning results [1] and are described in Table I. The length of the first and the second undulators (U1 and U2) is the total longitudinal distance including the magnetic length and the break length between undulator sections. In the first undulator, the FEL is operated in the exponential gain regime to avoid the growth of the energy spread due to FEL saturation. At the same time, the x-ray power after monochromator has to be at least two orders larger than the shot noise power. These considerations determine the length of the first undulator. The FEL in U2 has to reach saturation to reduce the fluctuations on the x-ray energy. 
TABLE I. Main parameters for the two-bunch self-seeding scheme.

\begin{tabular}{|c|c|c|c|c|}
\hline Parameter & Symbol & Uniform beam & LCLS beam & Unit \\
\hline X-ray photon energy & $\hbar \omega$ & 8 & 8 & $\mathrm{keV}$ \\
\hline X-ray wavelength & $\lambda_{0}$ & 1.55 & 1.55 & $\AA$ \\
\hline Electron energy & $E$ & 13.4 & 13.4 & $\mathrm{GeV}$ \\
\hline Bunch charge & $Q$ & 250 & 250 & $\mathrm{pC}$ \\
\hline Peak current & $I_{p}$ & 3 & 3 & $\mathrm{kA}$ \\
\hline Slice energy spread & $\sigma_{E}$ & 1.4 & 1.4 & $\mathrm{MeV}$ \\
\hline Slice emittance & $\gamma \epsilon_{0}$ & 0.6 & $\sim 0.4$ & $\mu \mathrm{m}$ \\
\hline Electron bunch length (fw) & $T_{b}$ & 50 & 80 & fs \\
\hline Separation of two bunches & $\Delta \mathrm{T}$ & $\sim 10$ & $\sim 10$ & ns \\
\hline U1 length & $L_{1}$ & 52 & 60 & $\mathrm{~m}$ \\
\hline U2 length & $L_{2}$ & 72 & 72 & $\mathrm{~m}$ \\
\hline Bypass chicane & $R_{56}$ & $\sim 200$ & $\sim 200$ & $\mu \mathrm{m}$ \\
\hline Monochromator crystal $^{\mathrm{a}}$ & & Dia (133) & $\mathrm{Si}(113)$ & \\
\hline Bragg angle at $8 \mathrm{keV}$ & $\theta$ & 71.3 & 28.2 & deg \\
\hline Relative bandwidth at $8 \mathrm{keV}$ & $\Delta \omega / \omega$ & $5 \times 10^{-6}$ & $3 \times 10^{-5}$ & \\
\hline
\end{tabular}

${ }^{\mathrm{a}} \mathrm{Dia}$ is abbreviated for diamond, and Si for silicon.

\section{A. Uniform beam}

We first illustrate the method by using uniform electron bunches, i.e., the current and energy distribution along each bunch is uniform, and the wakefield effects from the undulator chamber are ignored. The main parameters are shown in Table I. Four diamond (133) crystals are used to make a chicanelike monochromator after U1. The reflectivity curve of diamond (133) at $8 \mathrm{keV}$ is shown in Fig. 3 (red solid line), and the relative bandwidth is $5 \times 10^{-6}$. After the SASE x-ray pulse is generated from the first bunch in U1, we multiple the SASE field by the reflectivity curve 4 times to represent the effect of the monochromator. Since diamond (133) has a bandwidth narrower than the typical width of the SASE spectral spikes, only a single

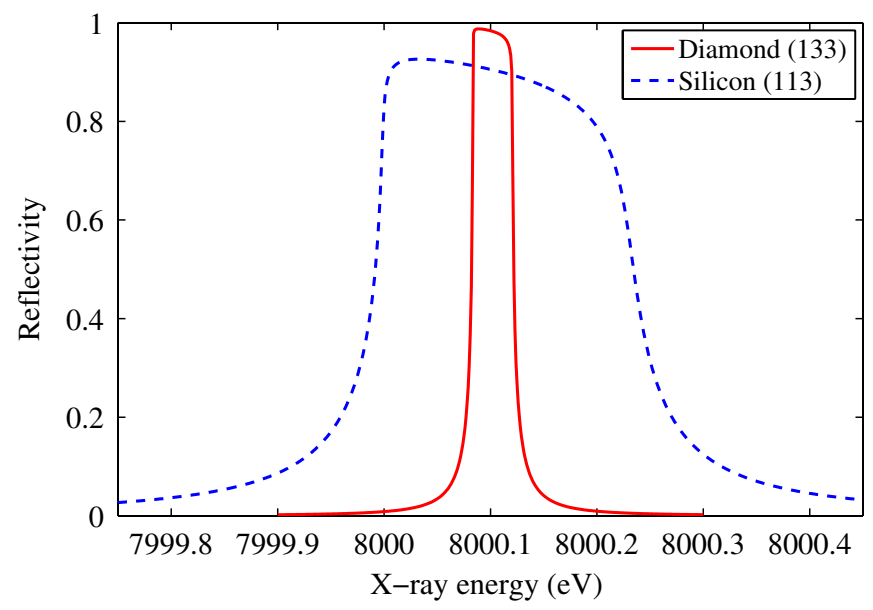

FIG. 3. (Color) The reflectivity curve of a single diamond (133) crystal (red solid line) and a single silicon (113) crystal (blue dashed line) around $8 \mathrm{keV}$ photon energy. The relative bandwidth is $5 \times 10^{-6}$ for diamond (133), and $3 \times 10^{-5}$ for silicon (113).
SASE spectral spike is selected. The filtered $\mathrm{x}$-ray pulse is stretched to about 80 fs (FWHM), and the relative x-ray energy fluctuation is at $100 \%$. Delayed by this monochromator by a path-length difference of $3 \mathrm{~m}$ relative to the electron bunch, the filtered $\mathrm{x}$-ray signal overlaps with the second electron bunch (arriving at the undulator about 10 ns later than the first bunch) and is amplified in U2 to saturation. The FEL gain curve in both $\mathrm{U} 1$ and $\mathrm{U} 2$ is shown in Fig. 4, which is based on an average of the x-ray power from 40 independent runs. The break between U1 and U2 is about $4 \mathrm{~m}$ long and can be used to install the weak electron-beam chicane and the crystal monochromator. Figure 5 shows a typical $\mathrm{x}$-ray power profile and its spectrum right after U2. Note that the bunch head is to the right in all figures related to time in this paper. We can see from the radiation power spectrum that the amplified seeding

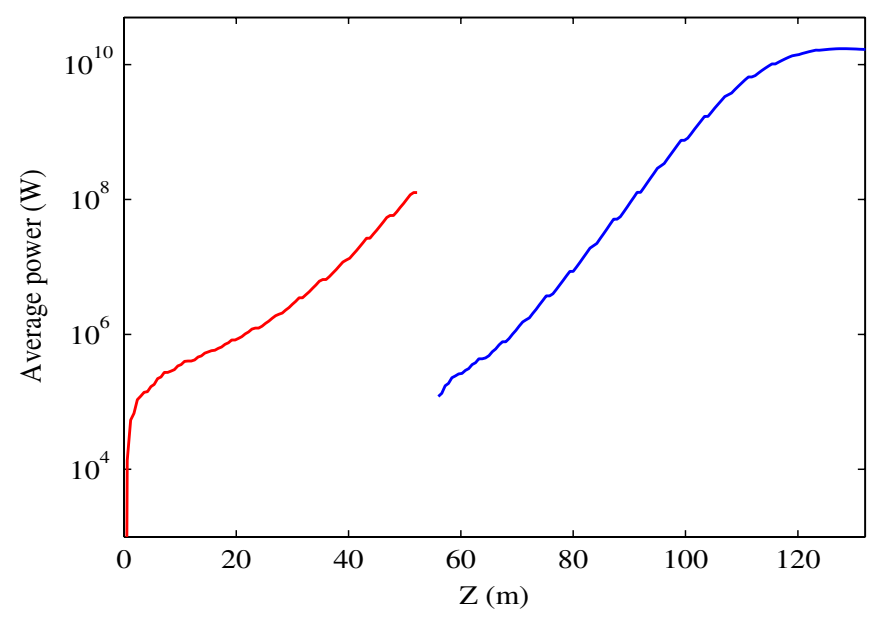

FIG. 4. (Color) Average FEL power in U1 (red curve) and U2 (blue curve) using a uniform beam, with a monochromator composed of four diamond (133) crystals between 52-56 m. 

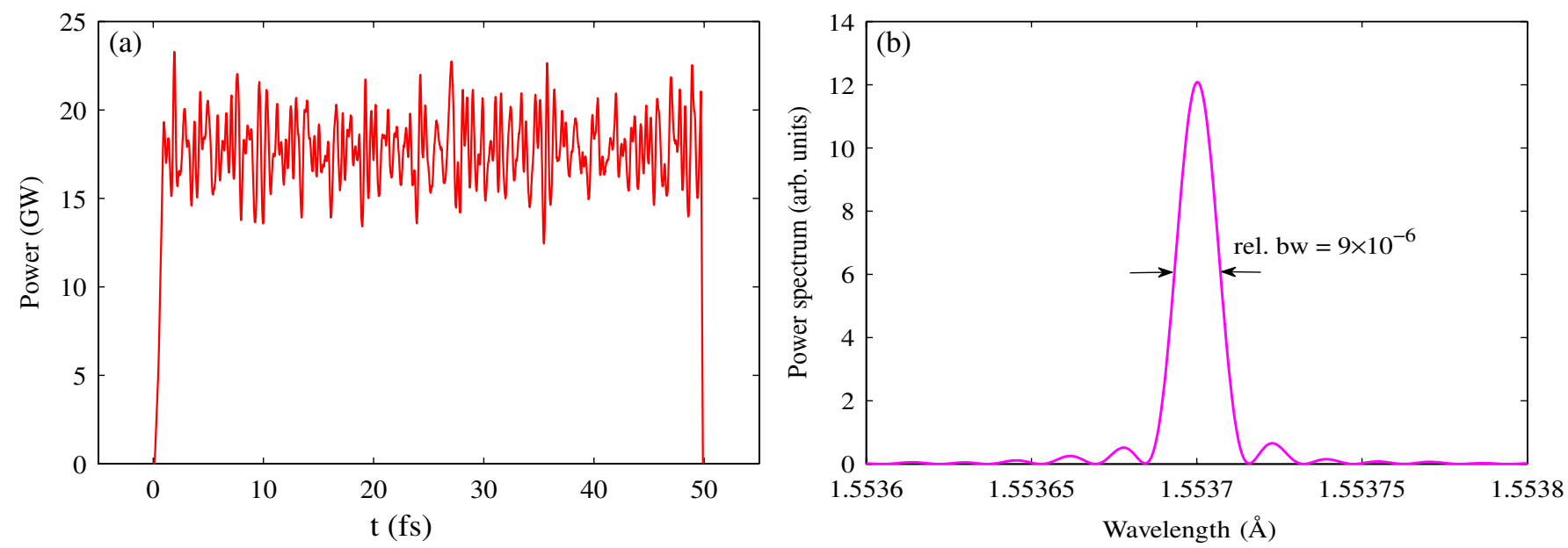

FIG. 5. (Color) A typical FEL power profile and spectrum after U2. The relative FWHM power spectrum bandwidth is about $9 \times$ $10^{-6}$. The bunch head is to the right in (a).

signal is much larger than the SASE background, and the relative bandwidth is about $9 \times 10^{-6}$ for the central spike. The statistical FEL energy fluctuation is reduced to $\sim 18 \%$ due to FEL saturation.

\section{B. LCLS beam}

A typical LCLS beam after acceleration and compression is usually not as simple as the case that we discussed for the uniform beam, especially for normal-conducting linacs where there are significant wakefield effects. For example, the LCLS beam operated at the undercompression mode has a double-horn current distribution, which can introduce additional energy modulation on the bunch due to wakefield effects in the undulator chamber. The bunch profile and the short-range resistive-wall energy loss in the LCLS undulator chamber [20] are shown in Fig. 6(a). This wake loss will introduce additional modu-

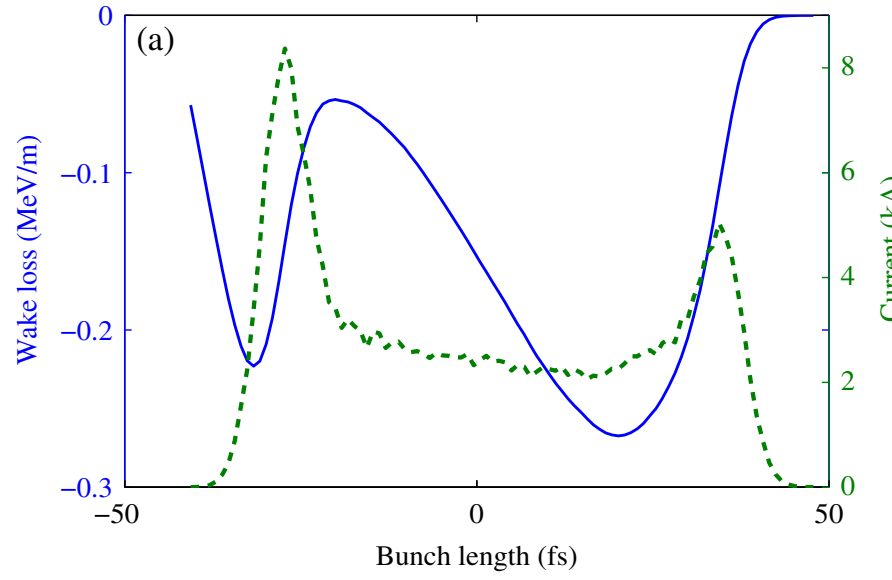

lation on the electron energy profile. We show the energy profiles at the entrance of U1 (blue curve) and U2 (red curve) in Fig. 6(b). The difference between the red and blue curves is from the wake loss in the undulator chamber. As a result, the seeded FEL bandwidth will be broadened due to this energy modulation. We address this effect in the following numerical studies.

Based on the LCLS start-to-end simulated beam with $250 \mathrm{pC}$ charge, we optimized the self-seeding setup by choosing the length of $\mathrm{U} 1$ and the monochromator materials after $\mathrm{U} 1$ and $\mathrm{U} 2$. The length of $\mathrm{U} 1$ in this case is $60 \mathrm{~m}$ (15 LCLS undulator sections). A four-dipole chicane with $R_{56}=200 \mu \mathrm{m}$ can occupy a total length of only $4 \mathrm{~m}$ (see Fig. 7). After passing through the chicane, the slice energy spread of the electron bunch $\left(1 \times 10^{-4}\right.$ at $\left.13 \mathrm{GeV}\right)$ is converted to a time shear through the chicane $R_{56}$, which is sufficient to wash out the noisy microbunching at $1.5 \AA$ developed in U1. The estimated collective effects (such as

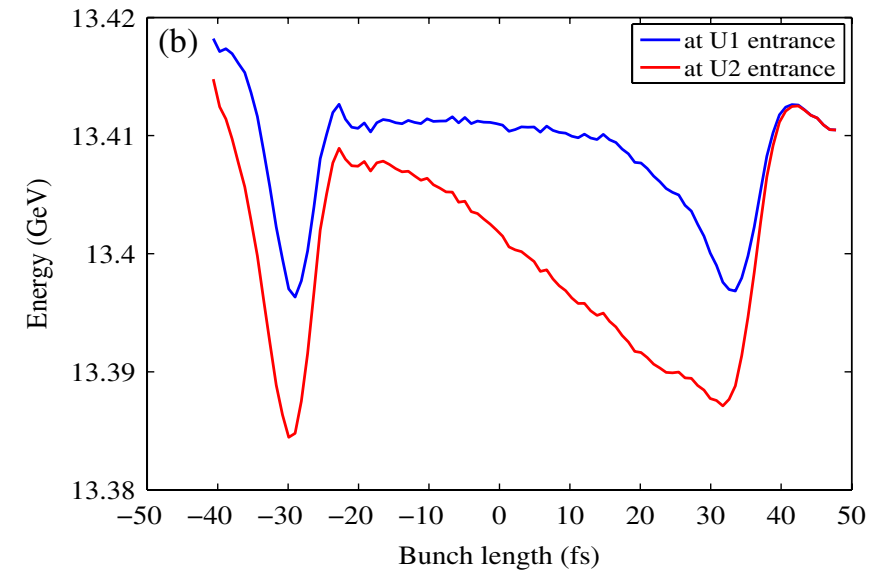

FIG. 6. (Color) (a) Simulated electron bunch current profile at the nominal LCLS operation (green dashed line) and wake loss (blue solid curve) from resistive wall of the undulator chamber. (b) The energy distribution at the entrance of the U1 and U2, and the difference is due to wake loss in $\mathrm{U} 1$. 


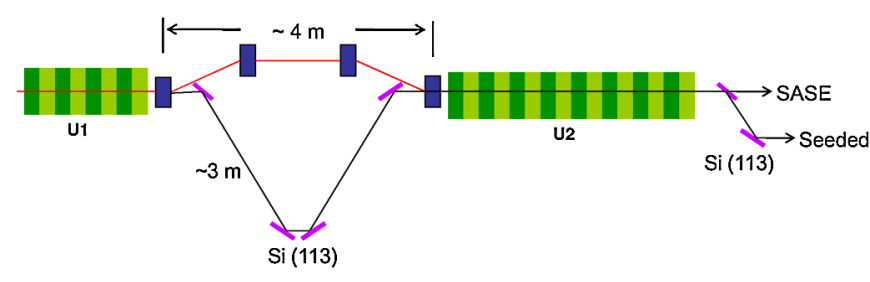

FIG. 7. (Color) Schematic of the two-bunch self-seeding scheme that may be implemented at the LCLS. The total x-ray delay introduced by the monochromator is about $3 \mathrm{~m}(10 \mathrm{~ns}$ ) (see Sec. III B for details).

coherent synchrotron radiation) in this bypass chicane is small, but further detailed studies will be done in the future. Silicon (113) is chosen here to form a four-bounce x-ray monochromator (see Fig. 7). Its reflectivity curve is shown in Fig. 3 (blue dashed line), with a net bandwidth of $\sim 3 \times 10^{-5}$ at $8 \mathrm{keV}$. This bandwidth is somewhat larger than the spectral width of the SASE spike, and is chosen to accommodate the wakefield effect in U2. Thus, a typical xray pulse after this monochromator still has a few temporal spikes as shown in Fig. 8.

We performed 30 statistical runs with simulated electron shot noise in U1. After passing the Si (113) monochromator, this narrow-bandwidth seed will be further amplified by the second electron bunch in $\mathrm{U} 2$. The power gain curve is shown in Fig. 9 from the two undulators. In Fig. 10, we show a typical power profile and FEL spectrum just following U2. Although the average power of the whole bunch shown in Fig. 9 still increases near the end of U2, the amplified seeding signals [the highlighted part in Fig. 10(a)] already reaches saturation by the end of $\mathrm{U} 2$. Compared with the ideal case, where almost all the electrons radiate within the seed bandwidth, here we have strong SASE signals outside this bandwidth due to the double-horn current profile and electron energy modula-

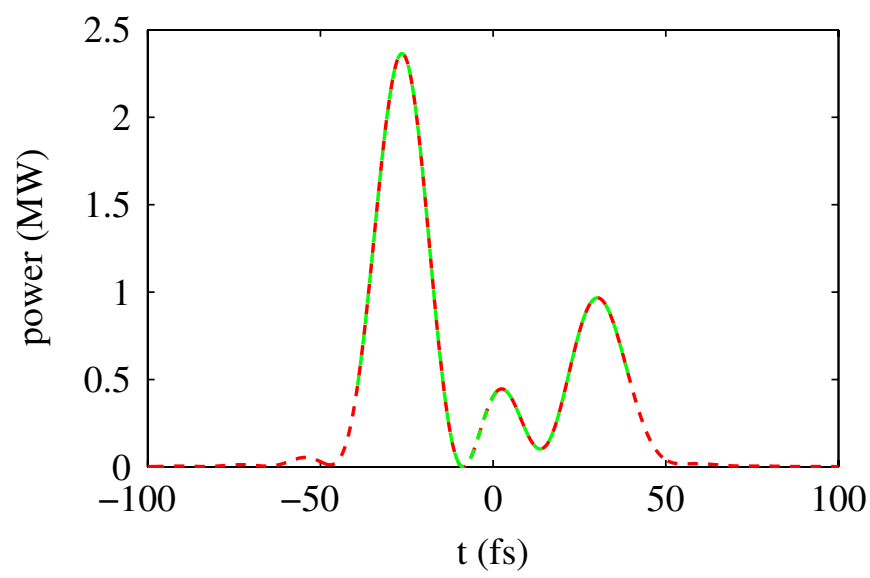

FIG. 8. (Color) A typical FEL power profile after U1 and silicon (113) monochromator based on the LCLS electron beam. This will be used as a seed in U2, and the green part represents the length of the electron bunch.

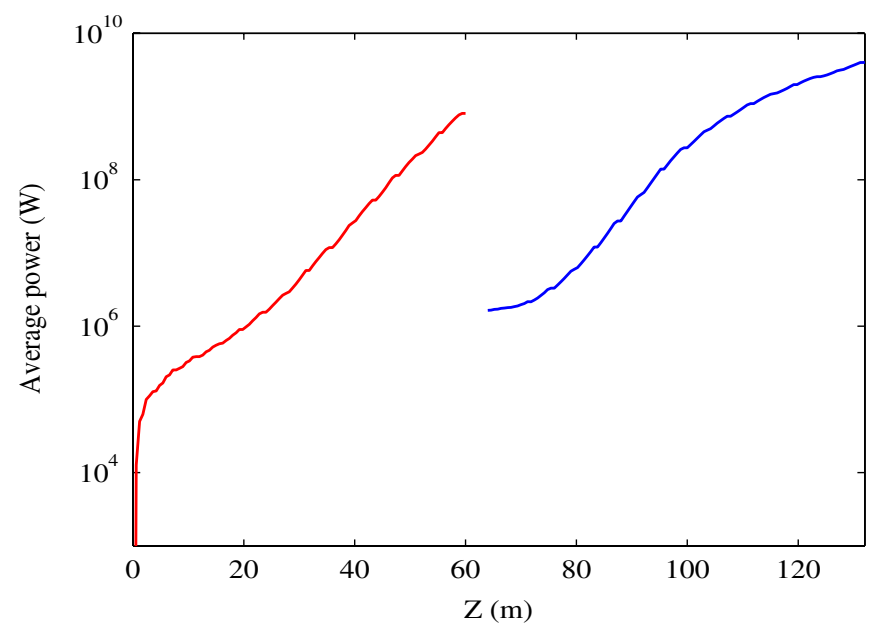

FIG. 9. (Color) Average FEL power in U1 (red curve) and U2 (blue curve) using a LCLS beam, with a monochromator composed of four silicon (113) between 60-64 m.

tion generated by the undulator wakefield. Nevertheless, we can use another pair of silicon (113) after U2 to filter out the SASE signal (see Fig. 7). As shown in Fig. 11(a), a fully coherent X-ray pulse with a peak power of $3 \mathrm{GW}$ can then be generated with a FWHM of $\sim 18 \mathrm{fs}$ after a pair of silicon (113). Since the bandwidth is broadened due to the electron energy modulation, we can use a larger bandwidth monochromator here to obtain more power for the seeded pulse. For example, if we replace silicon (113) after U2 by silicon (111) (with a relative bandwidth $1.4 \times 10^{-4}$ at $8 \mathrm{keV}$ ), the filtered FEL power profile is then given by Fig. 11(b). The FWHM x-ray pulse duration is reduced to $10 \mathrm{fs}$, and the peak power is about $10 \mathrm{GW}$. The statistical fluctuation of the narrow-bandwidth $\mathrm{x}$-ray pulse energy at U2 length of $72 \mathrm{~m}$ is about $15 \%$ for the two cases showed in Fig. 11. For the LCLS SASE operation at the hard x-ray wavelength, the achieved peak power is $\sim 30 \mathrm{GW}$ with a FWHM bandwidth of $\sim 3 \times 10^{-3}$ [1]. Using a pair of silicon (111) after U2, here we obtained a peak power of $\sim 10 \mathrm{GW}$ with a FWHM bandwidth of $\sim 2.3 \times 10^{-5}$. Compared with the SASE operation mode, we can enhance the peak spectral brightness by a factor of $\sim 30$ in this selfseeding scheme.

In addition to the longitudinally coherent pulse, we also have two SASE pulses. One of them is the SASE radiation from the first bunch in U2, while the other is the leaked SASE signal from the second bunch after the first silicon crystal of the second monochromator. Figure 12 shows a typical leaked SASE power profile after one silicon (111) at the end of U2. Compared with Fig. 10(a), we can see the central spike amplified from the seed is taken out. The SASE pulses can be separated from the seeded pulse by the post-U2 monochromator as shown in Fig. 7.

Since the LCLS electron bunch develops an energy chirp by the undulator wakefield, only a fraction of the bunch radiates within the narrow bandwidth of the monochro- 

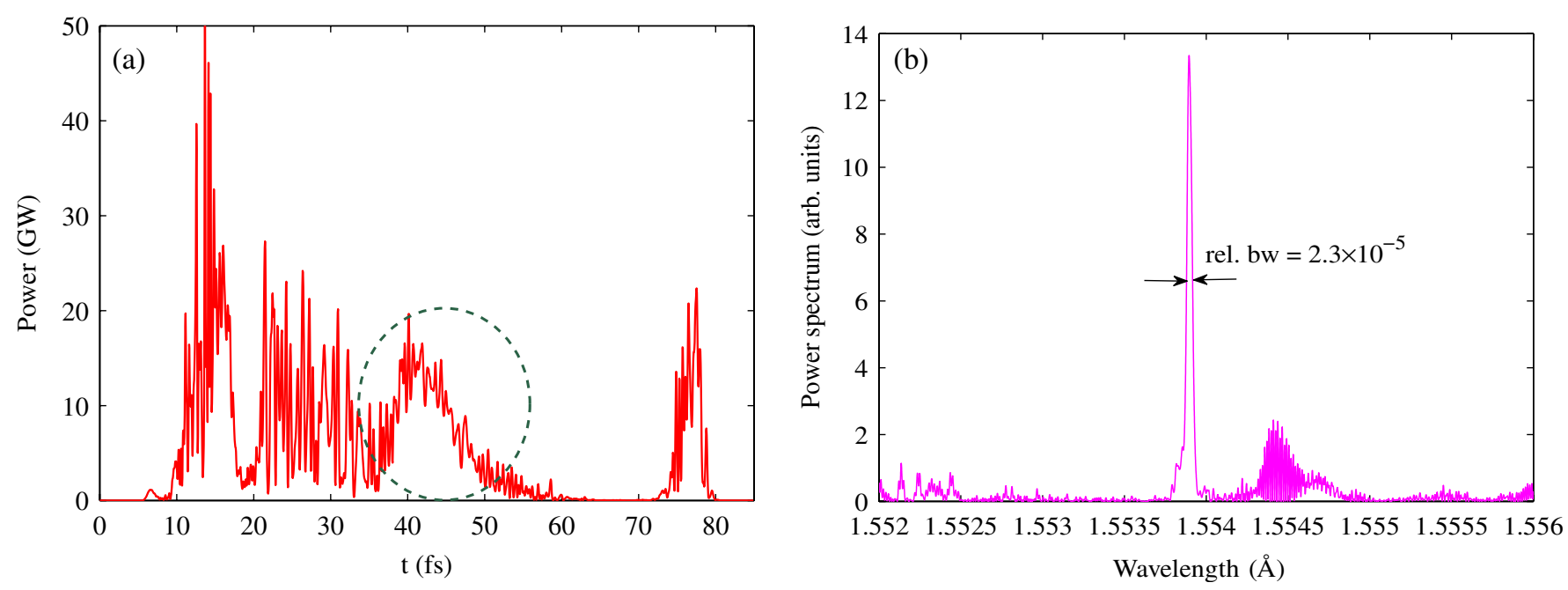

FIG. 10. (Color) A typical FEL power profile and spectrum right after U2 based on the LCLS electron bunch. The highlighted part with dashed line in (a) is the amplified signal from the seed. The power spectrum contains an amplified seed signal (with a relative bandwidth $\sim 2.3 \times 10^{-5}$ ) and a wider band SASE signal.
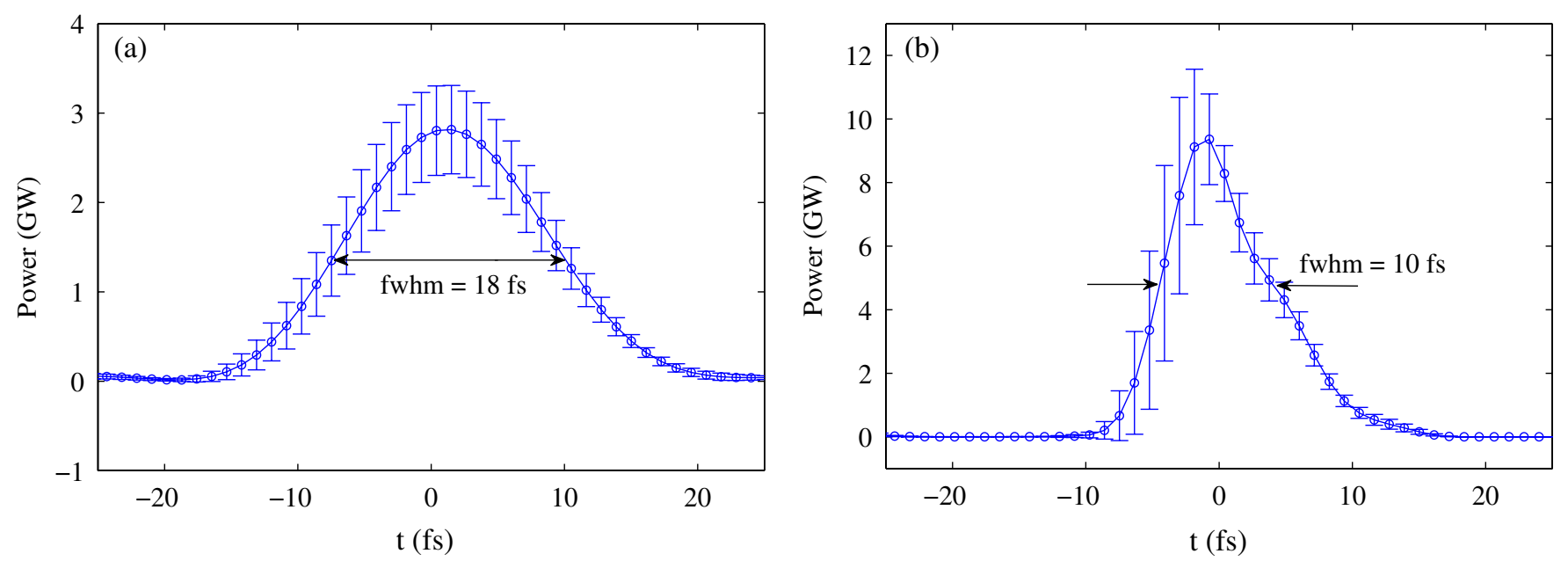

FIG. 11. (Color) The filtered FEL power profile after a pair of silicon (113) (a) and silicon (111) (b) at the end of U2. The vertical bars show the rms fluctuation level of the power.

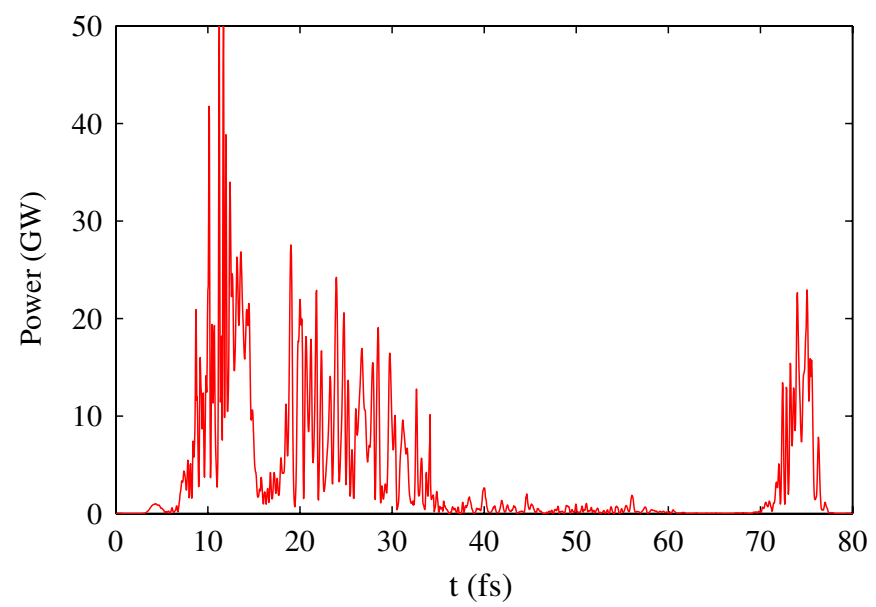

FIG. 12. (Color) A typical leaked out SASE power profile after passing through one silicon (111) crystal placed at the end of U2. matic seed. This leads to a shorter $\mathrm{x}$-ray pulse as shown above. In addition, the energy chirp may help reduce the seeded FEL energy fluctuations due to incoming electron energy jitter. An energy-chirped beam also leads to a slight shift of the FEL central wavelength at the end of U2 compared with the seeding wavelength due to a slight compression by $\mathrm{U} 2$. The shift in wavelength can be accounted for by slightly adjusting the angle of the postundulator monochromator.

In addition to the nominal operating charge of $250 \mathrm{pC}$, a low-charge mode of operation ( $20 \mathrm{pC}$ ) has been developed at the LCLS, which can produce x-ray pulses with duration less than 10 fs (FWHM) [21]. At this low charge, a typical hard x-ray pulse at $1.5 \AA$ contains about ten SASE spikes from simulations. We can also apply this method to the low-charge mode with silicon (111) monochromator to obtain a fully coherent, sub-10-fs hard x-ray pulse [15]. 
The wakefield effects from the linac and the undulator vacuum chamber are much reduced at such a low charge, although the timing requirement between the seed signal and the second low-charge bunch is more stringent.

\section{CONCLUSIONS}

The two-bunch self-seeding scheme proposed here takes advantage of two electron bunches to eliminate the timing constraint from the single-bunch self-seeding. Using an ideal electron bunch as well as a more realistic bunch from the LCLS start-to-end simulations, we demonstrate that the two-bunch self-seeding can produce a temporally coherent $\mathrm{x}$-ray pulse with the pulse duration in the range of 10-100 femtoseconds. This technique is perhaps the most straightforward type of seeding to use with hard x-ray FELs. It can also be easily incorporated in an existing SASE FEL facility with only a very modest amount of modifications to the machine layout. Finally, this method could be used with a train of bunches with each bunch seeding the one behind it. This would produce a train of narrow band, short $\mathrm{x}$-ray pulses at precise spacing.

\section{ACKNOWLEDGMENTS}

We thank K. Bane, F.-J. Decker, P. Emma, J. Frisch, $\mathrm{W}$. White, and $\mathrm{J}$. Wu for many useful discussions. This work was supported by Department of Energy Contract No. DE-AC02-76SF00515.

[1] P. Emma et al., in Proceedings of the 23rd Particle Accelerator Conference, Vancouver, Canada, 2009 (IEEE, Piscataway, NJ, 2009).
[2] J. Arthur et al., Linac Coherent Light Source (LCLS) Conceptual Design Report No. SLAC-R-593, Stanford, 2002 [see also http://ssrl.slac.stanford.edu/lcls/CDR/].

[3] SCSS XFEL Conceptual Design Report, Riken Harima Institute, Hyogo, Japan, 2005, edited by T. Tanaka and T. Shintake [see also http://www-xfel.spring8.or.jp].

[4] DESY Report No. DESY 2006-097, DESY, Hamburg, 2006, edited by M. Altarelli et al. [see also http://xfel. desy.de].

[5] B. D. Patterson et al., New J. Phys. 12, 035012 (2010).

[6] L. H. Yu, Phys. Rev. A 44, 5178 (1991).

[7] G. Stupakov, Phys. Rev. Lett. 102, 074801 (2009).

[8] D. Xiang and G. Stupakov, Phys. Rev. ST Accel. Beams 12, 030702 (2009).

[9] J. Feldhaus et al., Opt. Commun. 140, 341 (1997).

[10] E. Saldin et al., Nucl. Instrum. Methods Phys. Res., Sect. A 475, 357 (2001).

[11] C. Schroeder et al., J. Opt. Soc. Am. B 19, 1782 (2002).

[12] Z. Huang and R. Ruth, Phys. Rev. Lett. 96, 144801 (2006).

[13] K.-J. Kim, Y. Shvydko, and S. Reiche, Phys. Rev. Lett. 100, 244802 (2008).

[14] V. Miltchev et al., in Proceedings of FEL2006, Berlin, Germany, 2006 [http://accelconf.web.cern.ch/AccelConf/ f06/PAPERS/MOPPH053.PDF].

[15] G. Geloni, V. Kocharyan, and E. Saldin, DESY Report No. 10-033, 2010.

[16] C. Siders et al., Appl. Opt. 37, 5302 (1998).

[17] J. T. Seeman et al., SLAC Report No. SLAC-PUB-5438, 1991.

[18] O. Grimm et al., in Proceedings of the 10th European Particle Accelerator Conference, Edinburgh, Scotland, 2006 (EPS-AG, Edinburgh, Scotland, 2006).

[19] S. Reiche, Nucl. Instrum. Methods Phys. Res., Sect. A 429, 243 (1999).

[20] K. Bane and G. Stupakov, in Proceedings of the 21st Particle Accelerator Conference, Knoxville, 2005 (IEEE, Piscataway, NJ, 2005).

[21] Y. Ding et al., Phys. Rev. Lett. 102, 254801 (2009). 